\title{
Statistical Control Approach for Sleep-Mode Operations in IEEE $802.16 \mathrm{~m}$ Systems
}

\author{
Chung-Hsien Hsu, Member, IEEE, Kai-Ten Feng, Member, IEEE, and Chung-Ju Chang, Fellow, IEEE
}

\begin{abstract}
The power-saving class of type I (PSC I), which is one of the sleep-mode operations specified in the IEEE 802.16e standard, is designed to reduce power consumption for nonrealtime traffic. However, the inefficiency of the PSC I comes from the configuration of its operation and the utilized mechanism of binary-exponential traffic detection. Based on the concepts of IEEE $802.16 \mathrm{~m}$ sleep-mode operation, a statistical sleep window control (SSWC) approach is proposed to improve the energy efficiency of a mobile station (MS) with nonreal-time downlink traffic in this paper. The SSWC approach constructs a discrete-time Markov-modulated Poisson process (dMMPP) for representing the states of nonreal-time traffic. Furthermore, a partially observable Markov decision process (POMDP) is exploited within the SSWC approach to conjecture the present traffic state. Based on the estimated traffic state and the considerations of tolerable delay and/or queue size, two suboptimal policies, including the sleep ratio-based (SR) and energy cost-based (EC) policies, are proposed within the SSWC approach. The efficiency of the proposed SSWC approach is evaluated and compared via simulations. Simulation results show that the proposed SSWC approach outperforms the conventional IEEE 802.16e PSC I and the evolutional PSC I of the IEEE $802.16 \mathrm{~m}$ system in terms of both energy conservation and packet delay.
\end{abstract}

Index Terms-IEEE $802.16 \mathrm{e}$, IEEE $802.16 \mathrm{~m}$, nonreal-time traffic, power saving, sleep mode.

\section{INTRODUCTION}

$\mathbf{T}$ HE IEEE 802.16-2004 standard [1] for broadband wireless networks (BWNs) has been developed to support various demands for high capacity, high data rate, and advanced multimedia services. The IEEE 802.16e amendment [2] enhances the original standard by addressing the issues of mobility management and energy conservation for mobile stations (MSs). With the support of mobility, the IEEE 802.16e technology can be applied to both vehicular and cellular-based networks to provide various types of services, e.g., file transfer, Internet access, and Voice over Internet Protocol [3], [4]. To

Manuscript received August 4, 2009; revised January 13, 2010 and April 24, 2010; accepted July 16, 2010. Date of publication August 26, 2010; date of current version November 12, 2010. This work was supported in part by the Aiming for the Top University and Elite Research Center Development Plan, by the National Science Council under Grant NSC 98-2221-E-009-065 and Grant NSC 99-2628-E-009-005, by the MediaTek Research Center at National Chiao Tung University, by the Universal Scientific Industrial Co., and by the Telecommunication Laboratories at Chunghwa Telecom Co. Ltd., Taiwan. The review of this paper was coordinated by Prof. H. Hassanein.

C.-H. Hsu is with the Industrial Technology Research Institute, Hsinchu 300, Taiwan (e-mail: stanleyhsu@itri.org.tw).

K.-T. Feng and C.-J. Chang are with the Department of Electrical Engineering, National Chiao Tung University, Hsinchu 300, Taiwan (e-mail: ktfeng@mail.nctu.edu.tw; cjchang@cc.nctu.edu.tw).

Color versions of one or more of the figures in this paper are available online at http://ieeexplore.ieee.org.

Digital Object Identifier 10.1109/TVT.2010.2070086 support future advanced services with higher data rate and higher mobility, the next generation of IEEE 802.16 systems is being developed within the IEEE $802.16 \mathrm{~m}$ task group [5]. Since mobility is considered to be a key feature in these systems, how to prolong the battery lifetime of MSs has been recognized as one of the critical issues. There are existing power-saving mechanisms specified in the IEEE 802.16e standard, whereas improved operations for energy conservation are also considered to be desirable for the IEEE $802.16 \mathrm{~m}$ system.

In the IEEE 802.16e standard, sleep mode is defined as a state in which an MS conducts prenegotiated periods of absence from the serving base station (BS) air interface and consequently reduces the power consumption. In the sleep mode, the MS is provided with a series of alternate sleep and listening windows. The sleep window is a time period in which the BS shall not transmit data or management messages to the MS. During the listening window, on the other hand, the MS is expected to transmit/receive data or management messages based on the same manner as in the state of normal operation. Three types of sleep-mode operation, which correspond to power-saving classes (PSCs), are specified in the standard for supporting different types of traffic. The main difference among these PSCs is the way to determine the length of sleep windows. The binaryexponential increment of sleep window length is provided by the PSC of type I (PSC I) to support delay-tolerant nonreal-time traffic, e.g., File Transfer Protocol, Hypertext Transfer Protocol, and e-mail services. Real-time traffic with a guaranteed service rate such as audio and video services is targeted by the PSC of type II (PSC II), wherein the length of the sleep window is constant. The PSC of type III is only utilized for multicast or management traffic, which is considered to be a supplementary PSC to occasionally deliver control packets. Owing to the unpredictability nature of nonreal-time traffic, existing research in [6]-[14] has been conducted to extensively study the performance of the PSC I for energy conservation. On the other hand, based on the quality-of-service (QoS) requirements defined for real-time traffic, the design and modeling of the PSC II with the constant sleep window size becomes straightforward. In the case that there is mixed real-time and nonreal-time traffic within an MS, the constant length of sleep windows will still be utilized to guarantee the QoS requirements of real-time traffic [15], [16]. Therefore, as was interested and investigated by most of the existing research work, how to provide enhanced powersaving mechanisms for the PSC I with nonreal-time traffic will be the major concern in this paper.

For an MS with nonreal-time downlink traffic (i.e., traffic from the BS to the MS), an alternation of normal and sleep modes is provided for the MS to conserve its energy. The MS 
enters into the sleep mode while it has been idle for a prespecified period of time in the normal mode without data transmission. The sleep-mode operation of the MS is recommended to follow the PSC I, wherein the length of the sleep window starts with a predefined initial-window value and exponentially increases until a predefined final-window value is reached. If the arrival of packets is indicated by the BS within the fixedlength listening window, the MS deactivates the sleep mode and returns to the normal mode for data reception. The performance analyses of the PSC I have been investigated in recent research studies. The work in [6] and [7] analytically modeled the basic operation of the PSC I with the consideration of sleep mode only, whereas a system consists of normal and sleep modes is analyzed in [8] and [9]. The complex model for multiple downlink and uplink traffic is modeled and is investigated in [10]. On the other hand, enhanced sleep-mode mechanisms for the PSC I have been proposed and analyzed in [11]-[14]. The work in [11] and [12] dynamically enlarges the length of the initial sleep window to reduce the total number of listening windows, whereas the adjustment of the initial and final sleep windows is investigated in [13] and [14]. From the analytical results of these studies, it can be perceived that the inefficiency of the PSC I comes from the configuration of sleep-mode operation and the mechanism of binary-exponential traffic detection. For the purpose of enhancing the energy efficiency of MSs, several notions are included in the IEEE $802.16 \mathrm{~m}$ system description document (SDD) [17], and an improved sleep-mode operation is required by the IEEE $802.16 \mathrm{~m}$ system [18].

In this paper, a statistical sleep window control (SSWC) approach, following the notions of IEEE $802.16 \mathrm{~m}$ sleep-mode operation, is proposed to maximize the energy conservation for an MS with nonreal-time downlink traffic. The proposed SSWC approach determines the length of each sleep window that is appropriate for the present traffic state with the consideration of tolerable delay and/or queue size. Since the states of nonreal-time traffic are difficult to be obtained, a traffic model construction (TMC) procedure and a traffic state estimation (TSE) process are presented in the SSWC approach. The TMC procedure models a discrete-time Markov-modulated Poisson process (dMMPP) based on the trace of nonreal-time traffic, whereas a partially observable Markov decision process (POMDP) is exploited to conjecture the present traffic state in the TSE process. Furthermore, performance evaluations of window selection made in each traffic state are conducted to indicate the proper length of each sleep window. Based on the properties of POMDP, two suboptimal policies for sleep window selection (SWS) are exploited in the SSWC approach, including the sleep ratio-based (SR) and energy cost-based (EC) policies. The efficiency of the proposed SSWC approach is evaluated and compared via simulations. In terms of energy efficiency and packet transmission delay, simulation results show that the proposed SSWC approach outperforms both the conventional PSC I and the evolutional PSC I from the IEEE $802.16 \mathrm{~m}$ system. It is worthwhile to mention that the proposed SSWC approach adaptively determines the length of each sleep window, whereas only the initial sleep window size is dynamically adjusted by the schemes proposed in [11] and [12].
The rest of this paper is organized as follows: Section II briefly describes the sleep-mode operation of IEEE 802.16e and IEEE $802.16 \mathrm{~m}$ systems. The traffic model construction and state estimation within the proposed SSWC approach, including the TMC and TSE procedures, are explained in Section III. Section IV describes two selection policies, i.e., the SR and EC policies, of the SSWC approach with the considerations of delay and/or queue size. Performance evaluation of the proposed SSWC approach is illustrated in Section V. Section VI draws the conclusions.

\section{SleEP-Mode Operations IN IEEE 802.16 Systems}

\section{A. IEEE 802.16e Sleep-Mode Operation}

In the IEEE 802.16e system, nonreal-time traffic is supported by the PSC I, wherein three major parameters, including length of the initial sleep window $T_{\min }$, length of the final sleep window $T_{\max }$, and length of the listening window, are negotiated between the BS and the MS in the normal mode. Fig. 1(a) illustrates the sleep-mode operation of the PSC I in the IEEE 802.16e system. The MS enters the sleep mode while it has been idle for a period without data transportation in the normal mode. This period is normally standardized and serves as a timer in practical implementation. When the PSC I is activated, it starts with a sleep window of length $T_{\mathrm{SW}_{1}}=T_{\min }$. After the sleep window, a listening window is provided for the MS to receive the traffic indication message MOB-TRFIND, which is broadcasted from the BS. This message indicates whether there has been traffic addressed to the MS. If negative indication is acquired from the MOB-TRF-IND message, the MS will continue in the sleep mode after the listening window; otherwise, it will return to the normal mode for data reception. In the case that the MS continues in the sleep mode, the length of the next sleep window is doubled from the previous one, e.g., $T_{\mathrm{SW}_{2}}=2 \times T_{\mathrm{SW}_{1}}$. This process is repeated as long as the length of sleep window does not exceed $T_{\max }$. As the length of the sleep window reaches $T_{\max }$, the lengths of subsequent sleep windows will remain constant at $T_{\max }$. On the other hand, if the sleep mode of the MS is reactivated in the normal mode, the length of the first sleep window is reset as $T_{\min }$, e.g., $T_{\mathrm{SW}_{4}}=T_{\min }$, as shown in Fig. 1(a).

As can be observed in Fig. 1(a), a series of alternate sleep and normal modes are provided for the MS by adopting the PSC I in the IEEE 802.16e system. It is perceived that the inefficiency of the PSC I comes from two parts: 1) frequent state transitions and 2) underutilized listening windows. According to the operations of the PSC I, the MS deactivates the sleep mode and returns to the normal mode for data reception. The sleep mode is reactivated while an idle period without data transmission occurs in the normal mode. This configuration results in unnecessary energy waste of the MS during the idle periods. On the other hand, a binary-exponential mechanism for increasing the length of the sleep window is exploited in the PSC I to detect incoming traffic. This mechanism resets the length of the sleep window as $T_{\min }$ while the PSC I is reactivated, which consequently introduces a large number of underutilized listening windows. All the listening windows 

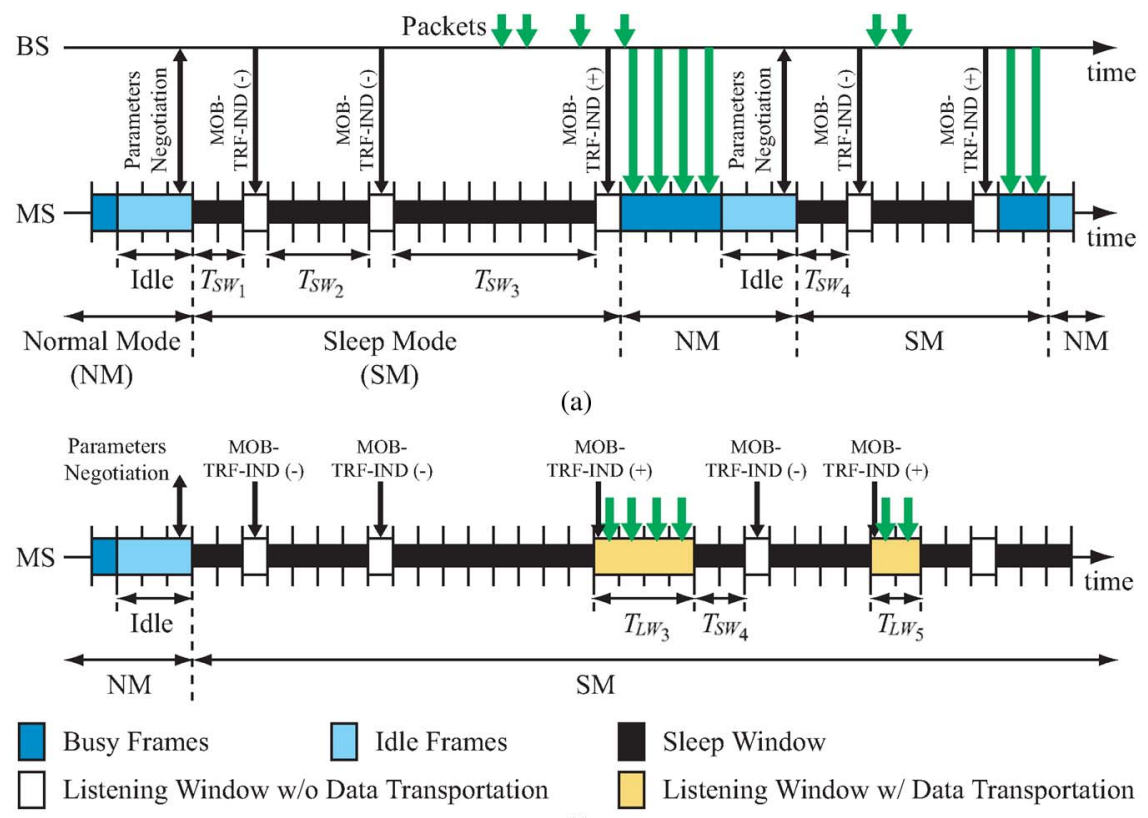

(b)

Fig. 1. Schematic of the sleep-mode operation in IEEE 802.16 systems. (a) PSC I in the IEEE 802.16e system. (b) Evolutional PSC I in the IEEE $802.16 \mathrm{~m}$ system.

are utilized to receive traffic indication messages only, which reduces energy efficiency of the MS.

\section{B. Notions of IEEE 802.16m Sleep-Mode Operation}

The IEEE $802.16 \mathrm{~m}$ standard for next-generation mobile BWNs is designed to support advanced services with higher data rate and higher mobility. Meanwhile, an improved powersaving mechanism is designed to enhance the energy conservation of MSs. To contend with aforementioned disadvantages of the PSC I and to provide a flexible sleep-mode operation, the IEEE 802.16m SDD specifies two significant notions.

1) Listening window adjustment. During listening windows, the MS is expected to transmit/receive data or management messages based on the same manner as in the state of normal operations. The length of each listening window can be dynamically adjusted based on traffic availability or control signaling.

2) Sleep-mode parameter update. The BS or the MS may dynamically update the parameters of sleep-mode operation (e.g., length of the sleep window) based on the change of traffic patterns. The updating procedure may be executed without deactivating the sleep mode.

The first notion successfully eliminates the energy waste resulted from the idle periods since data transportation is permitted during the adjustable listening windows in the sleep mode. As for the drawback of too many underutilized listening windows, the second notion provides a flexible platform to dynamically change the configuration of sleep-mode operation, which consequently reduces the number of underutilized listening windows.

Following the aforementioned notions, the PSC I with slight modification, named the evolutional PSC I, can be applied to the IEEE $802.16 \mathrm{~m}$ system. Fig. 1(b) illustrates the operation of the evolutional PSC I in the IEEE 802.16 m system. It can be observed that all the processes of the evolutional PSC I are the same as those of the conventional PSC I in the IEEE 802.16e system, as shown in Fig. 1(a), except for the period of data transportation. In the evolutional PSC I, the arriving packets will be received by the MS during the adjustable listening windows in the sleep mode, e.g., $T_{\mathrm{LW}_{3}}$ and $T_{\mathrm{LW}_{5}}$ in Fig. 1(b), instead of returning to the normal mode. After the completion of data transportation, the MS terminates the listening window and returns to the sleep state immediately. The length of the following sleep window is reset to the predefined initial-window value, e.g., $T_{\mathrm{SW}_{4}}=T_{\min }$. Therefore, the MS keeps staying in the sleep mode, and the process is repeated until an explicit termination of the sleep mode is requested by the MS or the BS. The evolutional PSC I successfully overcomes the problem of frequent state transitions; nevertheless, additional numbers of unutilized listening windows still exist within the sleep-mode operation. The performance of the evolutional PSC I will be evaluated and described in Section V.

\section{Proposed Statistical Sleep Window CONTROL APPROACH}

Based on the aforementioned notions of the IEEE $802.16 \mathrm{~m}$ system, there is motivation that a flexible sleep-mode operation should be provided to improve the energy consumption of MSs. The sleep-mode operation for an MS can be modeled as an SWS problem that is composed of multiple operations of control cycles. The definition of control cycle and the problem statement are described in the succeeding sections.

Definition 1 (Control Cycle): Given a BS and an MS that expects to enter the sleep mode or has stayed in the sleep mode, a control cycle $C_{i}$ is defined as a time duration consisting of a decision epoch $d_{i}$, a sleep window $\mathrm{SW}_{i}$, and a listening 


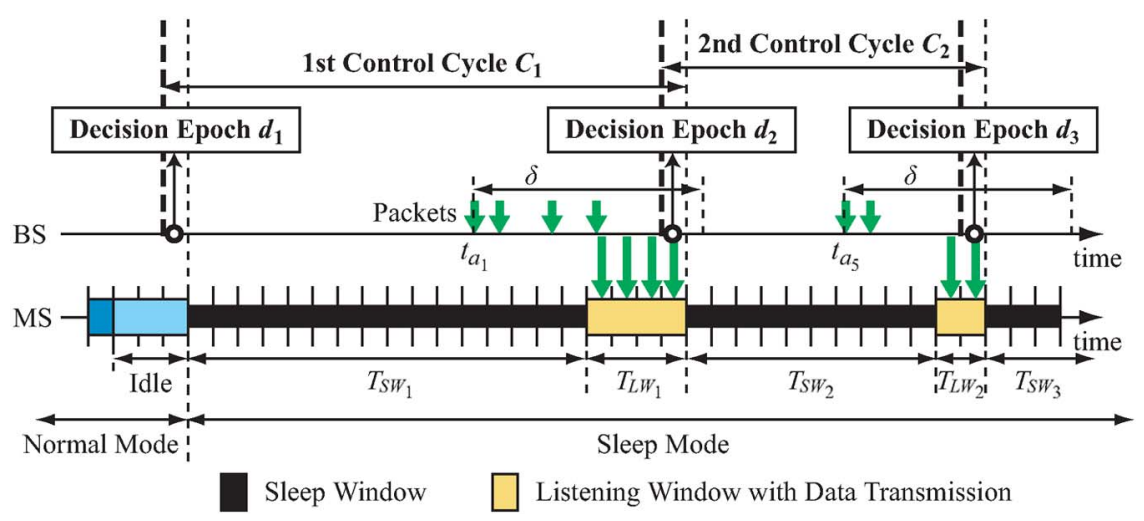

Fig. 2. Schematic of the ideal sleep-mode operation for an MS with nonreal-time downlink traffic.

window $\mathrm{LW}_{i}$. The BS determines the length of the sleep window $\mathrm{SW}_{i}$ at the decision epoch $d_{i}$. The MS stays in the sleep mode during the sleep window $\mathrm{SW}_{i}$ and wakes up for data transmission in the listening window $\mathrm{LW}_{i}$.

Problem 1 (SWS Problem): Given nonreal-time downlink traffic and a sequence of control cycles, how do we select a proper length of the sleep window under the tolerable packet delay $\delta$ for each control cycle to maximize the energy efficiency for the MS?

Fig. 2 depicts the time sequence of ideal sleep-mode operation for an MS with nonreal-time downlink traffic, wherein all the control signals are ignored for the convenience of description. It can be observed that each control cycle $C_{i}$ with $i \neq 1$ is overlapped with the adjacent control cycles $C_{i-1}$ and $C_{i+1}$. The first control cycle $C_{1}$ starts at the tail of MS's idle period in the normal mode. The remainder control cycles are individually executed at the end of the listening window within the previous control cycle, e.g., the cycle $C_{2}$ begins at the last frame of the listening window $\mathrm{LW}_{1}$.

To resolve the SWS problem, an SSWC approach is proposed, which determines the length of each sleep window that is appropriate for the present traffic state and delay constraint. Since the parameter update process of sleep-mode operation is specified in the IEEE $802.16 \mathrm{~m}$ standard, there is no additional control overhead for the BS to announce the length of each sleep window for an MS in the proposed SSWC approach. As shown in Fig. 2, the first packet arrives at the BS at time $t_{a_{1}}$, and the tolerable delay is $\delta$. Therefore, the termination of the sleep window $T_{\mathrm{SW}_{1}}$ that is determined at the decision epoch $d_{1}$ should fall within the time interval ranging from $t_{a_{1}}$ to $t_{a_{1}}+\delta$. Similarly, at the decision epoch $d_{2}$, the length of the second sleep window $T_{\mathrm{SW}_{2}}$ is determined according to the packet arrival time $t_{a_{2}}$ and the delay constraint $\delta$. It is intuitive that the length selected for each sleep window is dominated by the knowledge of the present traffic patterns, e.g., packet arrival time and packet arrival rate. However, this type of information is considered difficult to obtain for nonreal-time traffic. Only the number of buffered packets that arrived during the previous control cycle can provide information for potential estimation of the current traffic state. In the proposed SSWC approach, according to the information acquired from previous states, a POMDP [19], [20] is exploited to conjecture the present state of nonreal-time traffic at each decision epoch. In the succeeding two sections, two procedures of the proposed SSWC approach, including the TMC and TSE processes, will be presented. The TMC procedure models a dMMPP based on the trace information from nonreal-time traffic, whereas the POMDP formulation is conducted in the TSE procedure to estimate the current traffic state. Based on the present traffic state estimated via these two procedures, two suboptimal policies for solving the SWS problem are designed within the SSWC approach, which will be explained in Section VI.

\section{A. TMC Procedure}

In the proposed SSWC approach, nonreal-time downlink traffic is modeled as a dMMPP, which is considered more generic than the conventional Poisson model and is able to capture the characteristics of the Internet traffic [21]-[23]. While the notion of the dMMPP construction presented in [23] is exploited to capture the characteristics of self-similar traffic at multiple timescales, it can also be applied to calculate the states of nonreal-time traffic. Therefore, the objective of the TMC procedure is to establish an inferred dMMPP model for nonreal-time traffic, which will be utilized in the TSE process. It is noted that the dMMPP is equivalent to the MMPP in the continuous time domain; however, it is a more natural model for data processing owing to the number of data arrivals that happen in sampling intervals [23].

The dMMPP can be regarded as a Markov random walk where the increments in each interval have a Poisson distribution. The parameter within the Poisson distribution is considered a function of the state in the Markov chain. Thus, the $M$-state dMMPP can be represented by a transition probability matrix $\mathbf{P}$ of the Markov chain and a matrix $\boldsymbol{\Lambda}$ of Poisson arrival rate. These matrices are defined as

$$
\begin{aligned}
\mathbf{P} & =\left[\begin{array}{cccc}
p_{1,1} & p_{1,2} & \cdots & p_{1, M} \\
p_{2,1} & p_{2,2} & \cdots & p_{2, M} \\
\vdots & \vdots & \ddots & \vdots \\
p_{M, 1} & p_{M, 2} & \cdots & p_{M, M}
\end{array}\right] \\
\boldsymbol{\Lambda} & =\left[\begin{array}{cccc}
\lambda_{1} & 0 & \cdots & 0 \\
0 & \lambda_{2} & \cdots & 0 \\
\vdots & \vdots & \ddots & \vdots \\
0 & 0 & \cdots & \lambda_{M}
\end{array}\right]
\end{aligned}
$$




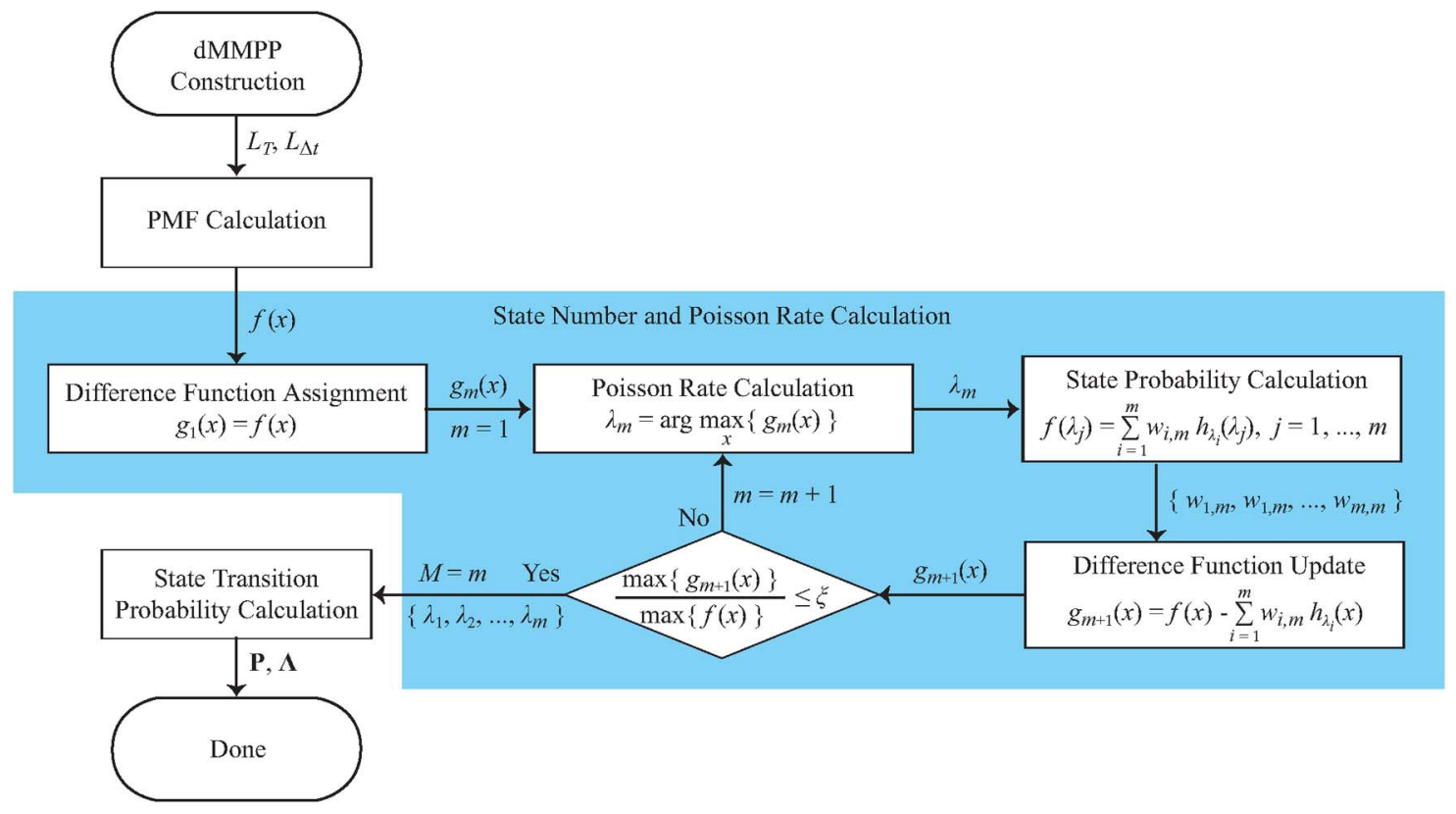

Fig. 3. Flow diagram of the TMC procedure.

where $p_{i, j}$ represents the transition probability from state $s_{i}$ to state $s_{j}$, and $\sum_{\forall j} p_{i, j}=1$ for $i, j \in\{1,2, \ldots, M\}$. The parameter $\lambda_{i}$ is the mean packet arrival rate in state $s_{i}$. All the parameters of the dMMPP are inferred from the probability mass function (PMF) of the number of arriving packets in a trace duration. Let $L_{T}$ and $L_{\Delta t}$ denote the lengths of trace duration and sampling interval, respectively. Fig. 3 depicts the flowchart of the TMC process, which is detailed in the succeeding paragraphs.

The procedure starts by computing the data sequence $\Upsilon=$ $\left\{\varrho_{1}, \varrho_{2}, \ldots, \varrho_{N}\right\}$, wherein each component $\varrho_{i}$ represents the number of arriving packets in the $i$ th $L_{\Delta t}$ and $N=L_{T} / L_{\Delta t}$. Based on the data sequence $\Upsilon$, the PMF of $x$ packets that arrived in time interval $L_{\Delta t}$ can be calculated as

$$
f(x)=\frac{\sum_{\forall \varrho_{i}=x} \varrho_{i}}{x N}, \quad i=1,2, \ldots, N
$$

The PMF $f(x)$ represents the distribution of the traffic, which can be treated as the weighted sum of all the Poisson arrival rates of the dMMPP model. Therefore, the states and the corresponding Poisson rates are obtained by iteratively subtracting a Poisson probability function from $f(x)$. Let $g_{m}(x)$ denote a function of the difference between $f(x)$ and the weighted sum of Poisson probability function at the $m$ th iteration, where $g_{1}(x)=f(x)$. In the $m$ th iteration, three steps are executed.

1) Poisson rate calculation. The $m$ th state of the dMMPP is acquired in the $m$ th iteration, wherein the corresponding Poisson rate is obtained as

$$
\lambda_{m}=\arg \max _{x}\left\{g_{m}(x)\right\} .
$$

2) State probability calculation. Based on the acquired Poisson rates $\left\{\lambda_{1}, \lambda_{2}, \ldots, \lambda_{m}\right\}$, the weight of each
Poisson probability function $\left\{w_{1, m}, w_{2, m}, \ldots, w_{m, m}\right\}$ is derived by solving the following set of linear equations:

$$
\left\{\begin{array}{c}
f\left(\lambda_{1}\right)=\sum_{i=1}^{m} w_{i, m} h_{\lambda_{i}}\left(\lambda_{1}\right) \\
f\left(\lambda_{2}\right)=\sum_{i=1}^{m} w_{i, m} h_{\lambda_{i}}\left(\lambda_{2}\right) \\
\vdots \\
f\left(\lambda_{m}\right)=\sum_{i=1}^{m} w_{i, m} h_{\lambda_{i}}\left(\lambda_{m}\right)
\end{array}\right.
$$

where $h_{\lambda_{i}}(x)$ denotes the Poisson probability function with rate $\lambda_{i}$.

3) Difference function update. The final step in the $m$ th iteration is to update the difference function as follows:

$$
g_{m+1}(x)=f(x)-\sum_{i=1}^{m} w_{i, m} h_{\lambda_{i}}(x) .
$$

The iterative procedure will be terminated when the ratio of the maximum of $g_{m+1}(x)$ to the maximum of $f(x)$ is lower than a predefined error threshold $\xi$, i.e., $\max \left\{g_{m+1}(x)\right\} /$ $\max \{f(x)\} \leq \xi$. Consequently, the number of states in the dMMPP is obtained as $M=m$, and the Poisson rate in each state $s_{i}$ is $\lambda_{i}$, where $i \in\{1,2, \ldots, M\}$.

The state transition probabilities of the constructed dMMPP are derived by counting the number of transitions between each pair of states. Therefore, each sampling interval will first be associated with one of the dMMPP states. Let $\psi_{j}(x)$ denote the probability of $x$ packets that arrived in time interval $L_{\Delta t}$ originating from state $s_{j}$, which can be obtained as

$$
\psi_{j}(x)=\frac{h_{\lambda_{j}}(x)}{\sum_{m=1}^{M} h_{\lambda_{m}}(x)} .
$$




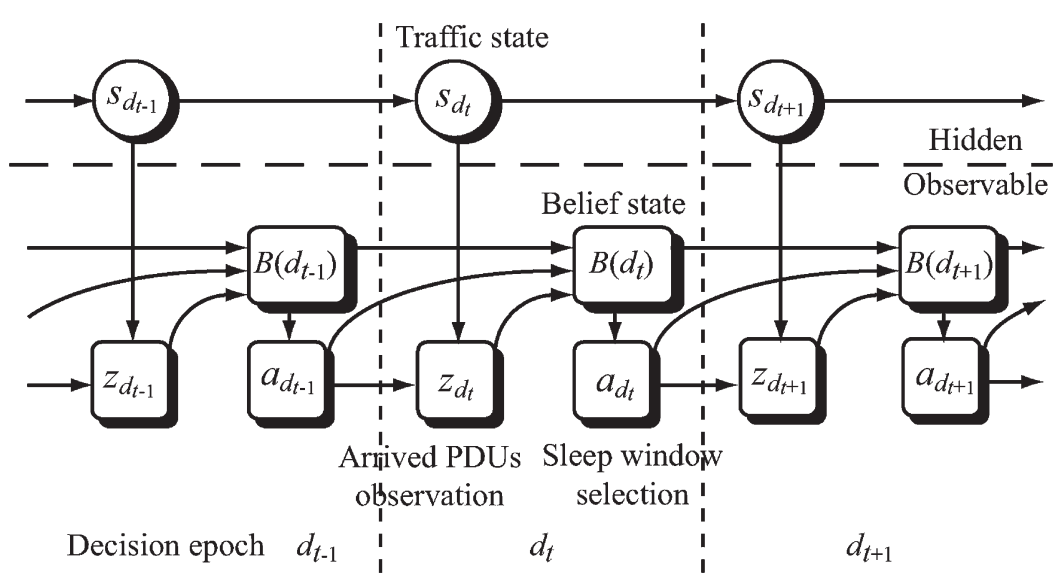

Fig. 4. Schematic of the POMDP model for the SSWC approach.

The state assignment for the $i$ th sampling interval is randomly calculated according to the probability set $\left\{\psi_{1}\left(\varrho_{i}\right)\right.$, $\left.\psi_{2}\left(\varrho_{i}\right), \ldots, \psi_{M}\left(\varrho_{i}\right)\right\}$. According to the state transition within the trace duration $L_{T}$, the transition probability of the dMMPP can be obtained as

$$
p_{i, j}=\frac{n_{i, j}}{\sum_{m=1}^{M} n_{i, m}}
$$

where $n_{i, j}$ represents the number of transitions from state $s_{i}$ to state $s_{j}$. A simple numerical example of the TMC procedure is given as follows: Considering a data sequence $\Upsilon=\{2,2,4$, $4,2,2\}$ with $N=6$, two states $s_{1}$ and $s_{2}$ with Poisson rates $\lambda_{1}=2$ and $\lambda_{2}=4$, respectively, can be calculated via (3)-(6) with the predefined error threshold $\xi=0.0001$. According to (7) and (8), the state transition probabilities $p_{1,1}=0.67, p_{1,2}=$ $0.33, p_{2,1}=0.5$, and $p_{2,2}=0.5$ can therefore be acquired. Based on the aforementioned construction process, the matrices $\mathbf{P}$ and $\boldsymbol{\Lambda}$ of the dMMPP can be acquired, which will be applied to the POMDP model in the succeeding section.

\section{B. TSE Procedure}

The TSE procedure exploits a POMDP model to conjecture the present traffic state at each decision epoch. A POMDP model is formally described as a tuple $\langle\mathcal{S}, \mathcal{A}, \mathcal{T}, \mathcal{Z}, \mathcal{O}, \mathcal{R}\rangle$, where $\mathcal{S}$ is a set of states, $\mathcal{A}$ is a set of actions, $\mathcal{T}$ is a set of state transition probabilities, $\mathcal{Z}$ is a set of observations, $\mathcal{O}$ is a set of observation probabilities, and $\mathcal{R}$ is a set of immediate rewards. For the proposed SSWC approach, $\mathcal{S}$ and $\mathcal{T}$ correspond to the set of dMMPP states and its state transition probability matrix $\mathbf{P}$, respectively, which are defined in the previous section. Since the objective of the SSWC approach is to resolve the SWS problem, the set of actions is defined as $\mathcal{A}=$ $\left\{a_{1}, a_{2}, \ldots, a_{N}\right\}$, where $a_{i}$ represents the action of selecting a sleep window of length $T_{a_{i}}$.

Considering a sequence of control cycle $\left\{C_{1}, C_{2}, \ldots, C_{T}\right\}$ in the proposed SSWC approach, the set of corresponding decision epochs is defined as $\mathcal{D}=\left\{d_{1}, d_{2}, \ldots, d_{T}\right\}$. Fig. 4 depicts the schematic of the POMDP model for the proposed SSWC approach. At each decision epoch $d_{t} \in \mathcal{D}$, the traffic state $s_{d_{t}} \in$ $\mathcal{S}$ is considered unobservable. However, the number of buffered packets that arrived during the previous control cycle $C_{t-1}$ can be observed and acquired. Thus, the set of observations is written as $\mathcal{Z}=\left\{z_{1}, z_{2}, \ldots, z_{Q}\right\}$, where $z_{i}$ denotes the number of packets that arrived in the interval between the $(i-1)$ th and the $i$ th decision epochs. Since the dMMPP model is utilized to capture the characteristics of the traffic source, the observation probability can be expressed as

$$
\begin{aligned}
o\left(a_{d_{t-1}}, s_{d_{t}}, z_{d_{t}}\right) & \triangleq \operatorname{Pr}\left(z_{d_{t}} \mid a_{d_{t-1}}, s_{d_{t}}\right) \\
& =\frac{\left(\lambda_{d_{t}} T_{a_{d_{t-1}}}\right)^{z_{t}}}{z_{d_{t}} !} e^{-\left(\lambda_{d_{t}} T_{a_{d_{t-1}}}\right)}
\end{aligned}
$$

which is a conditional probability of an observation $z_{d_{t}} \in \mathcal{Z}$ at decision epoch $d_{t}$ given the action $a_{d_{t-1}} \in \mathcal{A}$ made at $d_{t-1}$ and the present traffic state $s_{d_{t}} \in \mathcal{S}$ at $d_{t}$. It is intuitive that the length of the sleep window can be determined based on merely the latest observation of arriving packets. However, it has been shown in [19] that a nonoptimal solution is, in general, obtained within the POMDP model only based on the most recent observation.

To acquire the optimal policy, a belief state is introduced in the POMDP model for obtaining the history of previous observations. Given a decision epoch $d_{t} \in \mathcal{D}$, the set of belief states is defined as $\mathcal{B}\left(d_{t}\right)=\left\{b\left(s_{1}^{d_{t}}\right), b\left(s_{2}^{d_{t}}\right), \ldots, b\left(s_{M}^{d_{t}}\right)\right\}$, which represents the estimated probability distribution over the set of traffic states $\mathcal{S}=\left\{s_{1}, s_{2}, \ldots, s_{M}\right\}$. Each element $b\left(s_{i}^{d_{t}}\right)$ denotes the probability of traffic state $s_{i}$ at decision epoch $d_{t}$. It is noted that $0 \leq b\left(s_{i}^{d_{t}}\right) \leq 1, \forall s_{i} \in \mathcal{S}$ and $\sum_{\forall s_{i}} b\left(s_{i}^{d_{t}}\right)=1$, $\forall d_{t} \in \mathcal{D}$ since the traffic must reside in one of the states within the $\mathcal{S}$ set at any given decision epoch. As shown in Fig. 4, the belief state $\mathcal{B}\left(d_{t+1}\right)$ is updated at decision epoch $d_{t+1} \in \mathcal{D}$ by exploiting the previous action $a_{d_{t}} \in \mathcal{A}$ and the corresponding observation $z_{d_{t+1}} \in \mathcal{Z}$. Thus, each element $b\left(s_{i}^{d_{t+1}}\right)$ of the belief state $\mathcal{B}\left(d_{t+1}\right)$ can be derived as

$$
\begin{aligned}
& b\left(s_{i}^{d_{t+1}}\right) \\
& \quad=\operatorname{Pr}\left(s_{i}^{d_{t+1}} \mid \mathcal{B}\left(d_{t}\right), a_{d_{t}}, z_{d_{t+1}}\right) \\
& \quad=\frac{\operatorname{Pr}\left(z_{d_{t+1}} \mid s_{i}^{d_{t+1}}, a_{d_{t}}, \mathcal{B}\left(d_{t}\right)\right) \operatorname{Pr}\left(s_{i}^{d_{t+1}} \mid a_{d_{t}}, \mathcal{B}\left(d_{t}\right)\right)}{\operatorname{Pr}\left(z_{d_{t+1}} \mid a_{d_{t}}, \mathcal{B}\left(d_{t}\right)\right)}
\end{aligned}
$$




$$
=\frac{o\left(s_{i}^{d_{t+1}}, a_{d_{t}}, z_{d_{t+1}}\right) \sum_{s_{j}^{d_{t}} \in \mathcal{S}} p_{j, i} b\left(s_{j}^{d_{t}}\right)}{\sum_{s_{j}^{d_{t}} \in \mathcal{S}} \sum_{s_{i}^{d_{t+1}} \in \mathcal{S}} b\left(s_{j}^{d_{t}}\right) p_{j, i} o\left(s_{i}^{d_{t+1}}, a_{d_{t}}, z_{d_{t+1}}\right)} .
$$

Following the example described in the TMC procedure, the numerical results from the TSE procedure are presented as follows: From the previous section, two states $s_{1}$ and $s_{2}$ with Poisson rates $\lambda_{1}=2$ and $\lambda_{2}=4$, respectively, can be calculated associated with the state transition probabilities $p_{1,1}=0.67, p_{1,2}=0.33, p_{2,1}=0.5$, and $p_{2,2}=0.5$. Considering the action of selecting a sleep window size with one frame is determined at the decision epoch $d_{1}$ (i.e., $T_{a_{d_{1}}}=1$ ) and an observation of two frames is obtained at the next decision epoch $d_{2}$ (i.e., $z_{d_{2}}=2$ ), two observation probabilities $o\left(a_{d_{1}}, s_{1}^{d_{2}}, z_{d_{2}}\right)=0.2706$ and $o\left(a_{d_{1}}, s_{2}^{d_{2}}, z_{d_{2}}\right)=0.1464$ can therefore be calculated via (9). Let the set of belief states at $d_{1}$ be $\mathcal{B}\left(d_{1}\right)=\left\{b\left(s_{1}^{d_{1}}\right), b\left(s_{2}^{d_{1}}\right)\right\}=\{0.7,0.3\}$. According to (12), the probabilities of states $s_{1}$ and $s_{2}$ at $d_{2}$ can be computed as $b\left(s_{1}^{d_{2}}\right)=0.7502$ and $b\left(s_{2}^{d_{2}}\right)=0.2498$, respectively. Consequently, the set of belief states at $d_{2}$ can be obtained as $\mathcal{B}\left(d_{2}\right)=\left\{b\left(s_{1}^{d_{2}}\right), b\left(s_{2}^{d_{2}}\right)\right\}=\{0.7502,0.2498\}$. It is noted that the belief state is a summed statistics for the entire history of the process, which progressively incorporates the action and the corresponding observation at each decision epoch. Since the belief state is updated at each decision epoch, the time complexity is obtained as $O(|\mathcal{S}|)$, where $|\mathcal{S}|$ represents the total number of states in $\mathcal{S}$. Based on the belief state, more precise traffic states can be estimated by exploiting the proposed SSWC approach.

\section{Sleep Window Selection Policy}

In this section, two window selection policies of the SSWC approach are proposed and explained. Since the SSWC approach determines the length of each sleep window according to the present traffic state, the system performance of each state/action pair will first be investigated via different evaluation metrics in Section IV-A. Based on the evaluation results, as well as the considerations of tolerable delay and/or queue size, the suboptimal policies for resolving the SWS problem are proposed in the SSWC approach, as shown in Section IV-B.

\section{A. Evaluation Metrics}

Three evaluation metrics, including sleep ratio, energy cost, and packet delay, of each state/action pair $\left(s_{i}, a_{k}\right), \forall s_{i} \in \mathcal{S}$ and $\forall a_{k} \in \mathcal{A}$, are investigated in the proposed SSWC approach. All of these metrics are explained as follows.

1) Sleep Ratio: To measure the proportion of sleep windows to the entire operation time for an MS in the sleep mode, the sleep ratio is defined as the length of a sleep window compared with the corresponding control cycle duration. Thus, the expected sleep ratio of the $\left(s_{i}, a_{k}\right)$ pair can be expressed as

$$
\bar{R}\left(s_{i}, a_{k}\right)=\frac{E\left[T_{\mathrm{SW}}\left(s_{i}, a_{k}\right)\right]}{E\left[T_{\mathrm{SW}}\left(s_{i}, a_{k}\right)\right]+E\left[T_{\mathrm{LW}}\left(s_{i}, a_{k}\right)\right]}
$$

where $E\left[T_{\mathrm{SW}}\left(s_{i}, a_{k}\right)\right]$ and $E\left[T_{\mathrm{LW}}\left(s_{i}, a_{k}\right)\right]$ represent the expected lengths of the sleep window and the following listening window, respectively. Since the action $a_{k}$ is selected in state $s_{i}$, the length of the sleep window $E\left[T_{\mathrm{SW}}\left(s_{i}, a_{k}\right)\right]=T_{a_{k}}$, whereas $E\left[T_{\mathrm{LW}}\left(s_{i}, a_{k}\right)\right]$ can be derived as

$$
E\left[T_{\mathrm{LW}}\left(s_{i}, a_{k}\right)\right]=\left(N_{\lambda_{i}, a_{k}}^{\mathrm{SW}}+N_{\lambda_{i}, a_{k}}^{\mathrm{LW}}\right) E[U]
$$

where $N_{\lambda_{i}, a_{k}}^{\mathrm{SW}}$ and $N_{\lambda_{i}, a_{k}}^{\mathrm{LW}}$ represent the average numbers of arriving packets in the sleep and listening windows, respectively. Let $U$ be the random variable of service time for each packet, and let $E[U]=1 / \mu$ be the mean service time. By applying Little's theorem [24], $N_{\lambda_{i}, a_{k}}^{\mathrm{SW}}$ and $N_{\lambda_{i}, a_{k}}^{\mathrm{LW}}$ can be expressed as

$$
\begin{aligned}
& N_{\lambda_{i}, a_{k}}^{\mathrm{SW}}=\lambda_{i} T_{a_{k}} \\
& N_{\lambda_{i}, a_{k}}^{\mathrm{LW}}=\lambda_{i} E\left[T_{\mathrm{LW}}\left(s_{i}, a_{k}\right)\right] .
\end{aligned}
$$

By substituting (15) and $E[U]=1 / \mu$ into (14), the length of the listening window based on an action $a_{k}$ made in state $s_{i}$ can be obtained as

$$
E\left[T_{\mathrm{LW}}\left(s_{i}, a_{k}\right)\right]=\frac{\lambda_{i} T_{a_{k}}}{\mu-\lambda_{i}} .
$$

With $E\left[T_{\mathrm{SW}}\left(s_{i}, a_{k}\right)\right]=T_{a_{k}}$ and (16), the value of (13) can be acquired.

2) Energy Cost: To evaluate the power consumption for an MS in the sleep mode, the energy cost is defined as the average energy consumption per frame during a control cycle. Let $\varepsilon_{\mathrm{SW}}$ and $\varepsilon_{\mathrm{LW}}$ denote the energy consumption per frame within the sleep and listening windows, respectively. Moreover, the energy consumption of switching between the listening window and the sleep window is considered $\varepsilon_{S}$. The expected energy cost of the $\left(s_{i}, a_{k}\right)$ pair can be intuitively expressed as

$$
\begin{aligned}
\bar{E}\left(s_{i}, a_{k}\right) \\
\quad=\frac{2 \varepsilon_{S}+\varepsilon_{\mathrm{SW}} E\left[T_{\mathrm{SW}}\left(s_{i}, a_{k}\right)\right]+\varepsilon_{\mathrm{LW}} E\left[T_{\mathrm{LW}}\left(s_{i}, a_{k}\right)\right]}{E\left[T_{\mathrm{SW}}\left(s_{i}, a_{k}\right)\right]+E\left[T_{\mathrm{LW}}\left(s_{i}, a_{k}\right)\right]} .
\end{aligned}
$$

3) Packet Delay: Within the sleep window, the arriving packets are buffered at the BS and will be transmitted to the MS within the subsequent listening window. For the purpose of satisfying the QoS requirement in terms of delay constraint, packet delay is defined as the time duration between the instant that the packet arrived at the medium-access control layer in the BS and the instant that the packet is completely transmitted to the MS. Since the packet arrival follows the Poisson distribution in each state and the service rate is assumed to be a general distribution, the $M / G / 1$ queuing model is utilized to describe packet arrival and departure. The expected packet delay of the $\left(s_{i}, a_{k}\right)$ pair can be acquired as

$$
\bar{D}\left(s_{i}, a_{k}\right)=E\left[D_{\mathrm{SW}}\left(s_{i}, a_{k}\right)\right]+E\left[D_{\mathrm{LW}}\left(s_{i}, a_{k}\right)\right]+E[U]
$$

where $E\left[D_{\mathrm{SW}}\left(s_{i}, a_{k}\right)\right]$ represents the average remaining length of the sleep window for the packet, whereas the expected waiting time of the packet during the listening window is 
denoted by $E\left[D_{\mathrm{LW}}\left(s_{i}, a_{k}\right)\right]$. According to $M / G / 1$ with server vacation [25], $E\left[D_{\mathrm{SW}}\left(s_{i}, a_{k}\right)\right]$ can be obtained as

$$
E\left[D_{\mathrm{SW}}\left(s_{i}, a_{k}\right)\right]=\frac{E\left[T_{\mathrm{SW}}^{2}\left(s_{i}, a_{k}\right)\right]}{2 E\left[T_{\mathrm{SW}}\left(s_{i}, a_{k}\right)\right]}=\frac{T_{a_{k}}}{2} .
$$

On the other hand, based on Pollaczek-Khintchine mean value formula [24] and Little's theorem, $E\left[D_{\mathrm{LW}}\left(s_{i}, a_{k}\right)\right]$ can be derived as

$$
E\left[D_{\mathrm{LW}}\left(s_{i}, a_{k}\right)\right]=\frac{\lambda_{i} E\left[U^{2}\right]}{2\left(1-\rho_{i}\right)}
$$

where $\rho_{i} \triangleq \lambda_{i} / \mu$ denotes the traffic intensity. By substituting (19) and (20) into (18), the expected packet delay $\bar{D}\left(s_{i}, a_{k}\right)$ can therefore be obtained.

\section{B. Suboptimal Selection Policies}

According to the aforementioned performance evaluations, two selection policies, including SR and EC policies, are proposed in the SSWC approach. For the SR policy, the immediate reward of each state/action pair is defined as the aforementioned sleep ratio, whereas the energy cost is utilized as the immediate reward in the EC policy. The reward set $\mathcal{R}$ of the POMDP is calculated via the Reward Assignment Algorithm, as illustrated in Algorithm 1, where $\Phi$ indicates the type of policy, i.e., $\Phi=$ $S R$ and $\Phi=E C$ denote the SR and EC policies, respectively. The algorithm assigns the reward for each $\left(s_{i}, a_{k}\right), \forall s_{i} \in \mathcal{S}$ and $\forall a_{k} \in \mathcal{A}$, with the considerations of tolerable delay $\delta$ and/or queue size $Q$, which are explained here.

1) Tolerable delay consideration $(\phi=\tilde{d})$. With the consideration of delay constraint, the expected packet delay derived from (18) is utilized to exclude the infeasible action. As can be seen in lines 4-10 of Algorithm 1, if the expected delay of $\left(s_{i}, a_{k}\right)$ satisfies the tolerable delay $\delta$, the immediate reward $r\left(s_{i}, a_{k}\right)$ is assigned according to either (13) or (17). Otherwise, a predefined value $\bar{R}_{\text {min }}$ or $\bar{E}_{\max }$ is assigned to represent the cost for the SR or the EC policy, respectively.

2) Queue size consideration $(\phi=\tilde{q})$. On the other hand, the reward assigned with the consideration of queue size can be found in lines 13-19 of Algorithm 1. The average number of arriving packets obtained from (15) is considered as a criterion in this case, whereas all the other processes of reward assignment are the same as that mentioned in the case of tolerable delay consideration.

3) Both of the delay and queue considerations $(\phi=\tilde{b})$. In the case of considering both the tolerable delay and queue size, all the aforementioned processes are conducted to assign proper rewards for all state/action pairs, i.e., lines 3-20 of Algorithm 1.

Given the sets $\mathcal{S}$ and $\mathcal{A}$, Algorithm 1 is considered to be a tablelookup algorithm since $\bar{R}\left(s_{i}, a_{k}\right), \bar{R}_{\min }, \bar{E}\left(s_{i}, a_{k}\right)$, and $\bar{E}_{\max }$ can be calculated in advance. Therefore, the time complexity of the algorithm becomes $O(|\mathcal{S}||\mathcal{A}|)$, where $|\mathcal{S}|$ and $|\mathcal{A}|$ represent the number of states and the number of actions in $\mathcal{S}$ and $\mathcal{A}$, respectively.
Algorithm 1: Reward Assignment Algorithm

Input: $\mathcal{S}, \mathcal{A}$, tolerable delay $\delta$, queue size $Q$, policy type $\Phi$, and consideration type $\phi$

Output: set of immediate rewards $\mathcal{R}(\mathcal{S}, \mathcal{A})$

1 foreach $s_{i} \in \mathcal{S}$ do

2 foreach $a_{k} \in \mathcal{A}$ do

3 if $(\phi=\tilde{d})$ or $(\phi=\tilde{b})$ then

$4 \quad$ if $\bar{D}\left(s_{i}, a_{k}\right) \leq \delta$ then

5

6

7

8

9

10

11

12

13

14

15

16

17

18

19

20

21

22

$r\left(s_{i}, a_{k}\right) \leftarrow \bar{R}\left(s_{i}, a_{k}\right)$ for $\Phi=S R$

(or $r\left(s_{i}, a_{k}\right) \leftarrow \bar{E}\left(s_{i}, a_{k}\right)$ for $\Phi=E C$ )

else

$r\left(s_{i}, a_{k}\right) \leftarrow \bar{R}_{\text {min }}$ for $\Phi=S R$

(or $r\left(s_{i}, a_{k}\right) \leftarrow \bar{E}_{\max }$ for $\Phi=E C$ )

end

end

if $(\phi=\tilde{q})$ or $(\phi=\tilde{b})$ then

if $N_{\lambda_{i}, a_{k}}^{\mathrm{SW}}+N_{\lambda_{i}, a_{k}}^{\mathrm{LW}} \leq Q$ then

$r\left(s_{i}, a_{k}\right) \leftarrow \bar{R}\left(s_{i}, a_{k}\right)$ for $\Phi=S R$

(or $r\left(s_{i}, a_{k}\right) \leftarrow \bar{E}\left(s_{i}, a_{k}\right)$ for $\Phi=E C$ )

else

$r\left(s_{i}, a_{k}\right) \leftarrow \bar{R}_{\text {min }}$ for $\Phi=S R$

(or $r\left(s_{i}, a_{k}\right) \leftarrow \bar{E}_{\max }$ for $\Phi=E C$ )

end

end

end

end

The optimal policy of the SWS problem is unavailable owing to the reason of uncertain traffic states. However, due to the belief states of the POMDP model, the uncertain traffic states can be estimated. Moreover, the reward of an action made in a given traffic state is provided by the immediate reward set $\mathcal{R}$. Based on these two types of information, the suboptimal policies can be acquired via adopting a $T$-step value function in the SSWC approach. For the SR policy, the decision of window selection made at decision epoch $d_{t} \in \mathcal{D}$ can be acquired as

$$
\begin{aligned}
D_{d_{t}}^{S R}\left(b\left(s_{i}^{d_{t}}\right)\right) & =\arg V_{d_{t}}^{S R}\left(b\left(s_{i}^{d_{t}}\right)\right) \\
& =\arg \max _{a_{k}^{d_{t}} \in \mathcal{A}}\left[\Gamma_{d_{t}}\left(b\left(s_{i}^{d_{t}}\right)\right)\right]
\end{aligned}
$$

where

$$
\begin{aligned}
\Gamma_{d_{t}}\left(b\left(s_{i}^{d_{t}}\right)\right) & =\sum_{s_{i}^{d_{t}} \in \mathcal{S}} b\left(s_{i}^{d_{t}}\right) r\left(s_{i}^{d_{t}}, a_{k}^{d_{t}}\right) \\
& +\gamma^{d_{t+1}} \sum_{z_{j}^{d_{t+1}} \in \mathcal{Z}} \operatorname{Pr}\left(z_{j}^{d_{t+1}} \mid a_{k}^{d_{t}}, \mathcal{B}\left(d_{t}\right)\right) V_{d_{t+1}}\left(b\left(s_{i}^{d_{t+1}}\right)\right)
\end{aligned}
$$

with $r\left(s_{i}^{d_{t}}, a_{k}^{d_{t}}\right)$ selected as either $\bar{R}\left(s_{i}, a_{k}\right)$ or $\bar{R}_{\text {min }}$ according to Algorithm 1 for the SR scheme. The function $V_{d_{t}}^{S R}\left(b\left(s_{i}^{d_{t}}\right)\right)$ in (21) is defined as the $T$-step value function for the SR policy at a decision epoch $d_{t}$, which starts at $d_{t}$, and there are $T-1$ decision steps remaining. The first item of (22) denotes the 
reward for a belief state $b\left(s_{i}^{d_{t}}\right) \in \mathcal{B}\left(d_{t}\right)$. The expected reward of the future belief state $b\left(s_{i}^{d_{t+1}}\right) \in \mathcal{B}\left(d_{t+1}\right)$ is represented in the second item. The parameter $\gamma^{d_{t+1}}$ is denoted as a discount factor of the $d_{t+1}$ step for convergence control of the value function. In other words, the value function $V_{d_{t}}^{S R}\left(b\left(s_{i}^{d_{t}}\right)\right)$ intends to find the maximum sleep ratio of an action according to the currently estimated traffic state and the expected sleep ratio of future actions made in the successive states. On the other hand, for the EC policy, the decision made at $d_{t}$ and its corresponding $T$-step value function can be obtained as

$$
\begin{aligned}
D_{d_{t}}^{E C}\left(b\left(s_{i}^{d_{t}}\right)\right) & =\arg V_{d_{t}}^{E C}\left(b\left(s_{i}^{d_{t}}\right)\right) \\
& =\arg \min _{a_{k}^{d_{t}} \in \mathcal{A}}\left[\Gamma_{d_{t}}\left(b\left(s_{i}^{d_{t}}\right)\right)\right]
\end{aligned}
$$

where the function $\Gamma_{d_{t}}\left(b\left(s_{i}^{d_{t}}\right)\right)$ is defined in (22), with $r\left(s_{i}^{d_{t}}, a_{k}^{d_{t}}\right)$ selected as either $\bar{E}\left(s_{i}, a_{k}\right)$ or $\bar{E}_{\max }$ based on Algorithm 1 for the EC scheme. Unlike that for the SR policy, minimum reward is utilized in the value function $V_{d_{t}}^{E C}\left(b\left(s_{i}^{d_{t}}\right)\right)$ for the EC case to minimize the energy consumption of the MS.

\section{Performance Evaluation}

In this section, simulations are conducted to evaluate the performance of the proposed SSWC approach in comparison with the conventional PSC I and the evolutional PSC I. A single BS/MS pair with nonreal-time downlink traffic is considered as the simulation scenario. The simulation is implemented via a MATLAB event-driven simulator. All the required procedures and functions of both the BS and the MS for the sleep-mode operation have been implemented in the simulator. Specifically, the required parameter negotiation and update functions associated with the traffic indication mechanism are implemented in both the BS and the MS for the sleep-mode operation. Furthermore, the proposed TMC and TSE procedures and the window selection policies are only conducted in the BS to determine the feasible sleep window size for the MS. The parameters adopted within the simulation are listed in Table I, where the energy consumption parameters are acquired from the industrial manufactured mobile WiMAX chip [26]. Each simulation run is executed with 13 minutes, in which the MS stays in the normal mode during the first 3 minutes and starts the sleep-mode operation at the fourth minute. Each obtained result is the average of 100 simulation runs. For the proposed SSWC approach, the traffic trace of the first $3 \mathrm{~min}$ within the simulation is utilized to infer the number of traffic states, as described in Section III-A. Moreover, the three-step value function is considered in the simulation, wherein the discount factor $\gamma$ for the future $i$ th step is selected as $(0.5)^{i}$ for $i \in\{1,2\}$. It is represented that the rewards calculated later will have less value than an equivalent reward received closer to the present.

Fig. 5 illustrates the sleep-mode operations of the conventional PSC I (denoted IEEE 802.16e), the evolutional PSC I (denoted IEEE $802.16 \mathrm{~m}$ ), the SSWC approach with the SR policy (denoted SSWC-SR), and the SSWC approach with the EC policy (denoted SSWC-EC) under the traffic state of $\lambda=0.1$. The top subplot shows the arriving packets in frames
TABLE I

SIMULATION PARAMETERS

\begin{tabular}{|l|c|}
\hline Parameter & Value \\
\hline Frame duration & $5 \mathrm{~ms}$ \\
\hline Idle period (IEEE 802.16e) & 4 frames \\
\hline Default Listening Window length (IEEE $802.16 \mathrm{e} / \mathrm{m}$ ) & 1 frame \\
\hline Initial-Sleep Window length (IEEE $802.16 \mathrm{e} / \mathrm{m})$ & 1 frame \\
\hline Final-Sleep Window length (IEEE $802.16 \mathrm{e} / \mathrm{m})$ & 256 frames \\
\hline Sleep Window length (SSWC) & {$[1,256]$ frames } \\
\hline Energy consumption of busy frame & $280 \mathrm{~mW}$ \\
\hline Energy consumption of idle frame & $120 \mathrm{~mW}$ \\
\hline Energy consumption of sleep frame & $10 \mathrm{~mW}$ \\
\hline Energy consumption of state switch & $1 \mathrm{~mW}$ \\
\hline Mean service rate $(\mu)$ & 3 packets/frame \\
\hline
\end{tabular}

[37400, 37 700] of the simulation, whereas the corresponding operations of all the schemes are depicted in the remainder subplots. For each approach, the state of 1 denotes that the MS is awake in the normal mode or during the listening window of the sleep mode, whereas the state of 0 denotes that the MS stays in the sleep state. It can be observed that the MS spends lots of time in the awake state while the binary-exponential power-saving mechanism of IEEE $802.16 \mathrm{e}$ or IEEE $802.16 \mathrm{~m}$ is adopted. With the proposed SSWC approach, on the other hand, the MS mostly remains in the sleep state and only wakes up to receive the incoming traffic since each sleep period is determined according to the present traffic state estimated via the POMDP. The performance comparisons among these schemes are discussed in the succeeding sections.

\section{A. Effect of Delay Constraints}

The effect of delay constraints is discussed via the performance comparisons, as shown in Figs. 6-8. The performance of average packet delay between the two proposed SSWC approaches is illustrated in Fig. 6, where EC and SR denote the approaches of SSWC-EC and SSWC-SR, respectively. Due to the proposed reward assignment and accurate state estimate, the performance of all the schemes satisfies the respective delay constraints over various mean packet arrival rates. As for the cases that do not consider any delay constraints, the average packet delay is bounded by the maximum length of the sleep window, i.e., $640 \mathrm{~ms}$ for the window of length 256 frames in the simulation. The SSWC-EC approach has relatively higher packet delay than the SSWC-SR scheme resulted from the different definition of reward functions. For the reward that is defined as the sleep ratio (i.e., the SSWC-SR approach), the length of the sleep window that results in the highest reward value will be selected, whereas the window with the lowest reward value is chosen in the SSWC-EC approach to minimize the energy cost. These two policies result in different sleepmode operations, even if they can possess the same ratio of listening frames to sleep frames. For example, considering the duration of frames [37 543, 37 660], as shown in Fig. 5, there are three listening frames and 115 sleep frames that appeared in both schemes. In the SSWC-EC approach, one listening frame occurs at frame number 37600 , whereas two happen at numbers 37659 and 37660 . The SSWC-SR scheme, on the other hand, has all its three listening frames occurring at frame numbers 37558,37559 , and 37660 . Therefore, it can be observed that 

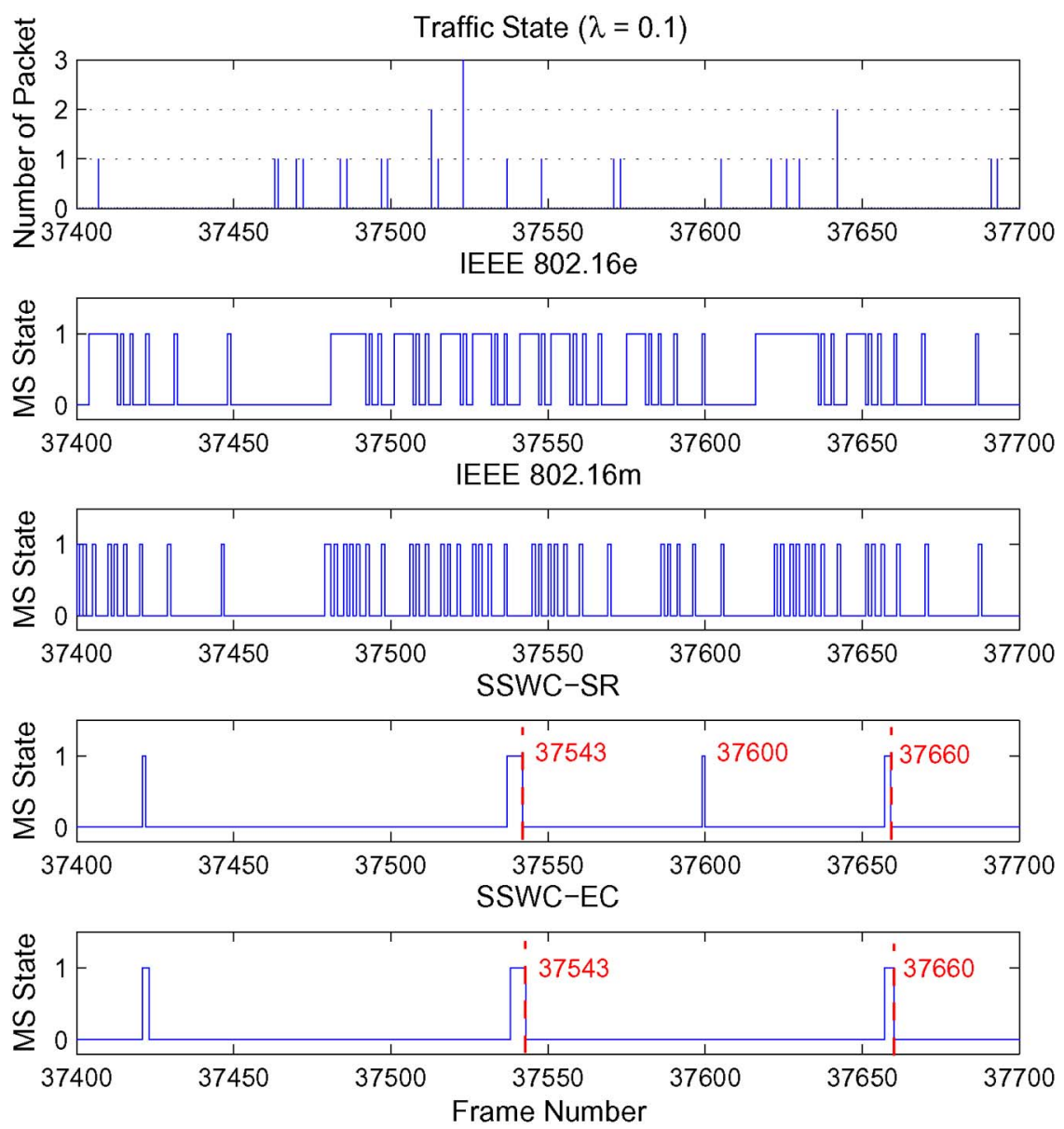

Fig. 5. Exemplified sleep-mode operation among IEEE 802.16 e, IEEE $802.16 \mathrm{~m}$, SSWC-SR, and SSWC-EC approaches under the traffic state of $\lambda=0.1$.

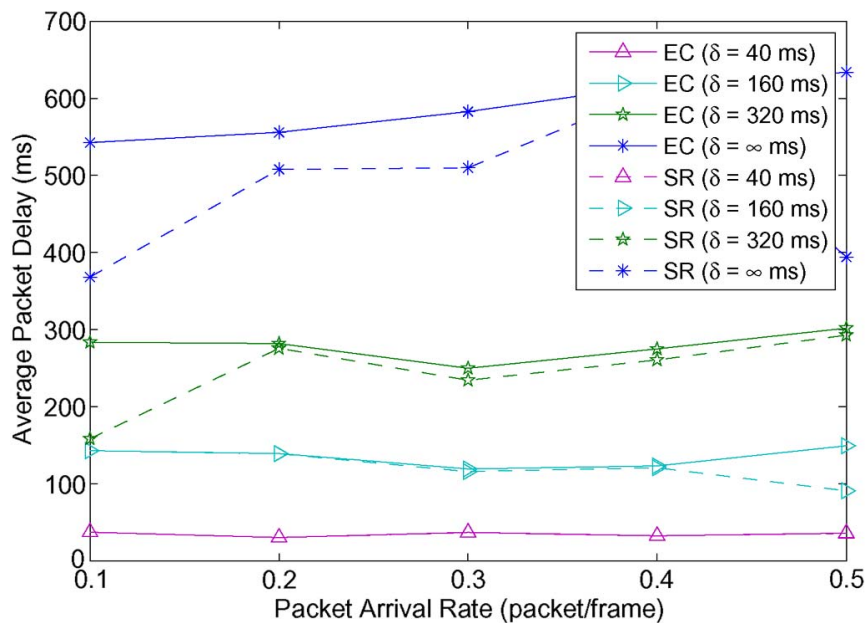

Fig. 6. Performance comparison of average packet delay between the two proposed SSWC approaches (i.e., EC and SR) with different delay constraints $\delta$.

both schemes result in the same sleep ratio of $115 / 118=0.975$ during this considered time interval. It is intuitive to see that the SSWC-EC approach intends to minimize the energy consumption such as to accumulate all the three listening frames to reduce the switching $\operatorname{cost} \varepsilon_{S}$ between listening and sleep windows. Nevertheless, the target of the SSWC-SR approach is to maximize the sleep ratio that results in the occurrence of the first listening frame at frame 37600 . The sleep ratio becomes

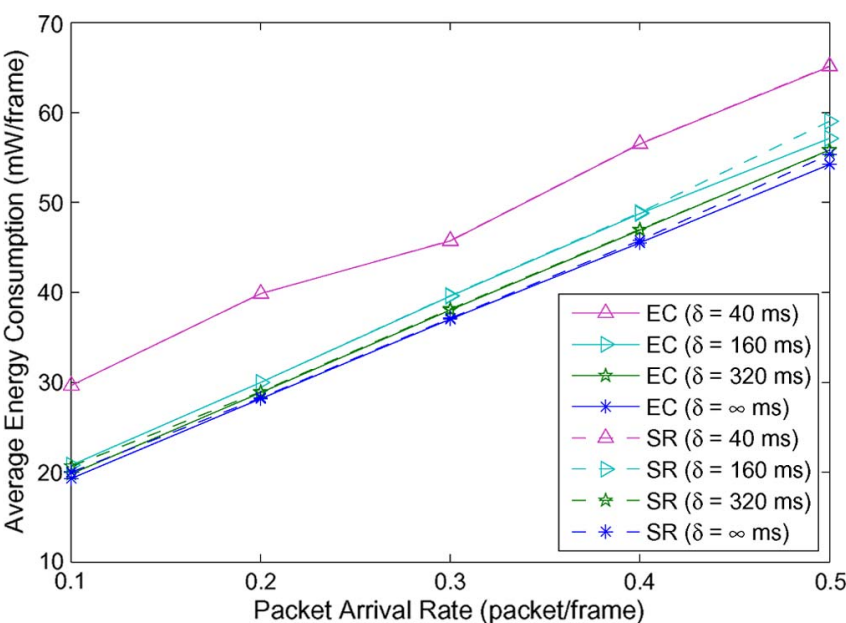

Fig. 7. Performance comparison of average energy consumption between the two proposed SSWC approaches (i.e., EC and SR) with different delay constraints $\delta$.

$57 / 58=0.983$ with the length of the sleep window equal to 57 frames and that of the listening window equal to one frame, which is higher than 0.975 by adopting the SSWC-EC scheme. Consequently, as shown in Fig. 6, the performance with lower packet delay can be obtained in the various cases by exploiting the SSWC-SR approach.

Fig. 7 depicts the performance comparison of average energy consumption between the two proposed SSWC approaches. It is 

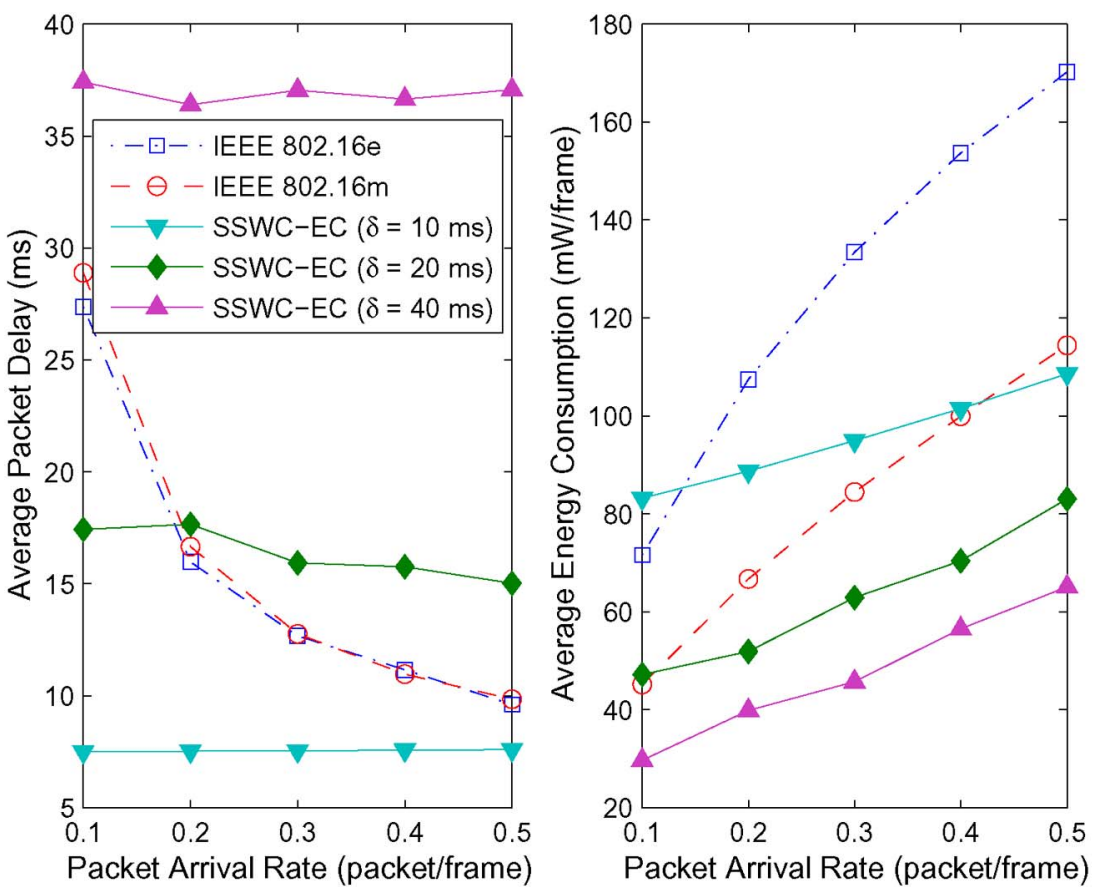

Fig. 8. Performance comparison among IEEE 802.16e, IEEE 802.16m, and SSWC-EC approaches with different delay constraints.

expected that the average energy consumption increases as the value of mean packet arrival rate is augmented in all the different cases. With lower tolerable delay, more energy consumption is observed in both of the proposed SSWC approaches. This can be attributed to the fact that the length of the sleep window is bounded by the delay constraint. In the sleep mode, the MS is provided with a series of alternate sleep and listening windows. For the purpose of satisfying tight tolerable delay, the shorter sleep windows will be selected, which incurs more number of listening windows during the simulation time and consequently increases the energy consumption. Comparing the SSWC-EC and SSWC-SR approaches, almost the same level of energy consumption is obtained since a similar ratio of listening frames to sleep frames exists in both schemes. However, the shorter sleep windows selected in the SSWC-SR scheme incurs more number of state transitions, i.e., from sleep state to awake state, and vice versa. Therefore, slightly higher energy consumption is shown in the SSWC-SR approach compared with that in the SSWC-EC scheme.

The performance comparisons among IEEE 802.16e, IEEE $802.16 \mathrm{~m}$, and the proposed SSWC approach over various packet arrival rates are shown in Fig. 8. Since similar performance is exhibited in the proposed SSWC-EC and SSWC-SR schemes with different packet delay considerations, the SSWCEC approach is utilized on behalf of the proposed SSWC scheme to compare with the conventional binary-exponential power-saving mechanisms. For the IEEE 802.16e and IEEE $802.16 \mathrm{~m}$ schemes, it is expected that the average packet delay will be decreased as the packet arrival rate is augmented (as in the left subplot of Fig. 8); meanwhile, the energy consumption is raised (as in the right subplot of Fig. 8). IEEE $802.16 \mathrm{~m}$ has slightly higher delay but a significant decrement of energy consumption compared with IEEE 802.16e, which can be attributed to the elimination of frequent state transitions. As mentioned in
Section II-B, in IEEE $802.16 \mathrm{~m}$, the MS terminates the listening window and returns to the sleep state immediately after the completion of data transportation. Without the existence of idle periods in IEEE $802.16 \mathrm{~m}$, energy conservation can be preserved. However, the opportunities to promptly receive the incoming traffic are dissolved and consequently incur packet transmission delay. Comparing between the proposed SSWCEC approach and the IEEE $802.16 \mathrm{~m}$ scheme, relatively better performance can be observed in the SSWC-EC approach. It can be seen that the energy consumption of the SSWC-EC $(\delta=20 \mathrm{~ms})$ approach is similar to that of IEEE $802.16 \mathrm{~m}$ at $\lambda=0.1$, but the SSWC-EC $(\delta=20 \mathrm{~ms})$ approach can provide significantly lower packet delay. It is owing to the reason that the SSWC approach determines the length of each sleep window according to the traffic state and delay constraint. Due to the same reason, as similar packet delays existed in both schemes, the SSWC approach will result in lower energy consumption compared with the IEEE $802.16 \mathrm{~m}$ scheme, e.g., the case with the SSWC-EC ( $\delta=20 \mathrm{~ms})$ scheme at $\lambda=0.2$.

\section{B. Effect of Queue Length Considerations}

Figs. 9-12 illustrate the effect of queue size considerations among the power-saving mechanisms. The performance comparisons of average packet delay among the proposed SSWC approaches are shown in Fig. 9. Given a traffic state, the larger queue size results in higher packet delay since the longer sleep window is selected either to maximize the sleep ratio in the SSWC-SR approach or to minimize the energy consumption in the SSWC-EC scheme. Since the number of arriving packets is increased with the augmentation of the packet arrival rate, the shorter sleep window is considered to satisfy the constant size of the queue, which consequently reduces the average packet delay. The SSWC-EC approach possesses higher packet delay 


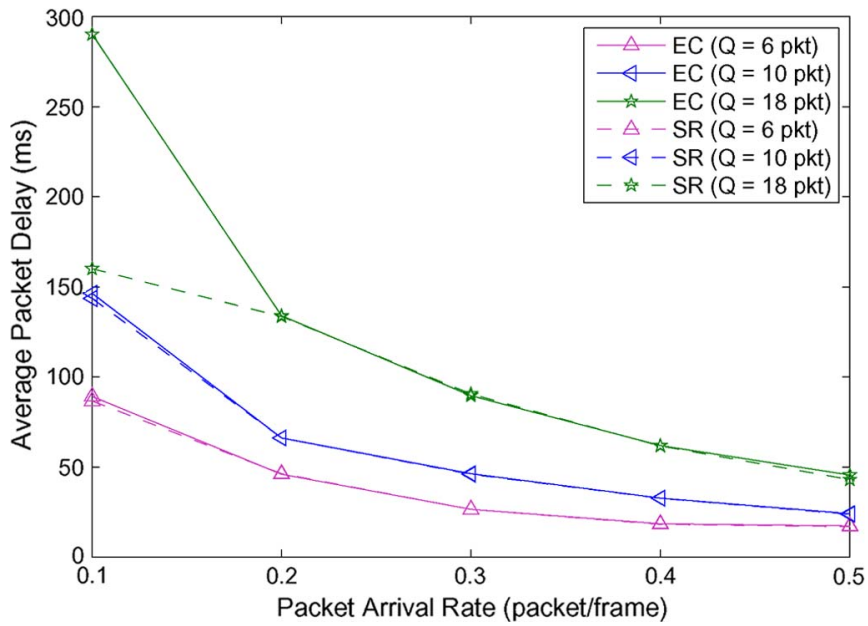

Fig. 9. Performance comparison of average packet delay between the two proposed SSWC approaches (i.e., EC and SR) with different queue size considerations $Q$.

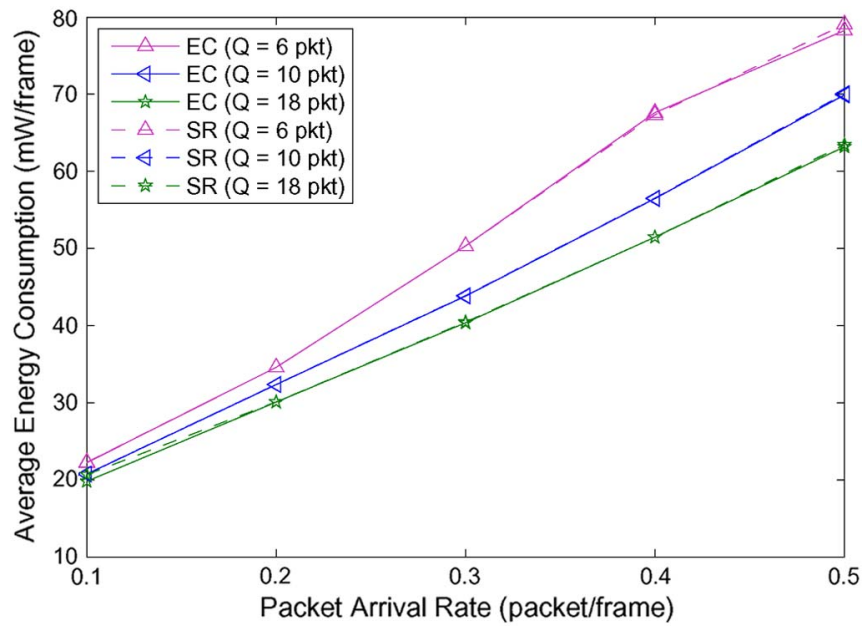

Fig. 10. Performance comparison of average energy consumption between the two proposed SSWC approaches (i.e., EC and SR) with different queue size considerations $Q$.

than the SSWC-SR scheme, which can be attributed to the same reason as explained in Section V-A.

Fig. 10 shows the performance of average energy consumption between the two proposed SSWC approaches. It is expected that the average energy consumption increases as the value of mean packet arrival rate is augmented in all the cases. Under the situations with smaller queue size, more energy consumption is required in the proposed SSWC approaches since the length of the sleep window will be limited by both the traffic state and the queue size. Considering the scenario of constant traffic state, shorter sleep windows will be selected with the consideration of smaller queue size, which results in an additional number of listening windows during the simulation time and, hence, increases the energy consumption. Owing to a similar ratio of listening frames to sleep frames, both the SSWC-EC and SSWC-SR approaches possess similar energy consumption, as shown in Fig. 10.

Fig. 11 illustrates the performance of packet overflow under various queue size considerations. Packet overflow is defined as the number of packets exceeding the predefined queue size compared with the total number of packets in the simulation. It is observed that both the IEEE 802.16e and IEEE $802.16 \mathrm{~m}$ schemes result in comparatively high packet overflow under the consideration of a queue size of less than five packets. This can be attributed to the fact that the bursty packets frequently incur overflowing packets, while the queue size is small. Packet overflow decreases as the queue size increases in both the IEEE 802.16e and IEEE 802.16m schemes since the large queue size provides the capability for enduring the bursty situation. On the other hand, the SSWC approaches possess relatively lower packet overflow owing to the reason that the length of each sleep window is determined according to the traffic state and considered queue size.

Performance comparisons among IEEE 802.16e, IEEE $802.16 \mathrm{~m}$, and the proposed SSWC-EC approach over various packet arrival rates are shown in Fig. 12. The SSWC-EC approach is adopted on behalf of the proposed SSWC scheme since both the EC and SR policies result in similar performance under the consideration of queue size. Compared with the IEEE 802.16e and IEEE 802.16m schemes, lower energy consumption can be acquired by adopting the SSWC approach, as shown in the right subplot of Fig. 12. The SSWC-EC approaches result in comparatively high packet delay (as in the left subplot of Fig. 12) since the length of each sleep window is selected according to the traffic state and considered queue size. The MS wakes up as the number of buffered packet is close to the considered queue length, which consequently increases the packet delay. Nevertheless, the primary objective of the proposed SSWC approach to reduce the energy consumption of the MS can still be achieved. The merits of the proposed schemes can be observed.

\section{CONCLUSIONS}

In this paper, an SSWC approach for the sleep-mode operation has been proposed to maximize the energy efficiency of an MS with nonreal-time downlink traffic. Following the notions of the IEEE $802.16 \mathrm{~m}$ system, the sleep-mode operation is formulated as an SWS problem. The SSWC approach selects the length of each sleep window based on the present traffic state and the considerations of tolerable delay and/or queue size. The TMC procedure of the SSWC approach formulates a dMMPP for nonreal-time downlink traffic via analyzing the traffic trace, which infers all the states of the traffic. Based on the constructed dMMPP, a POMDP is adopted in the TSE procedure of the SSWC approach to conjecture the present traffic state at each decision epoch. By exploiting the properties of the POMDP, two suboptimal policies, including the SR and EC policies, with the considerations of tolerable delay and/or queue size are proposed within the SSWC approach to resolve the SWS problem. The efficiency of the proposed SSWC approach is evaluated and compared via simulations. Simulation studies show that the proposed SSWC approach outperforms the conventional IEEE 802.16e PSC I and the evolutional PSC I of the IEEE $802.16 \mathrm{~m}$ system in terms of both energy efficiency and packet delay while the delay constraint is considered. On the other hand, the SSWC approach aggregates the buffered packets as much as possible while satisfying the predefined 

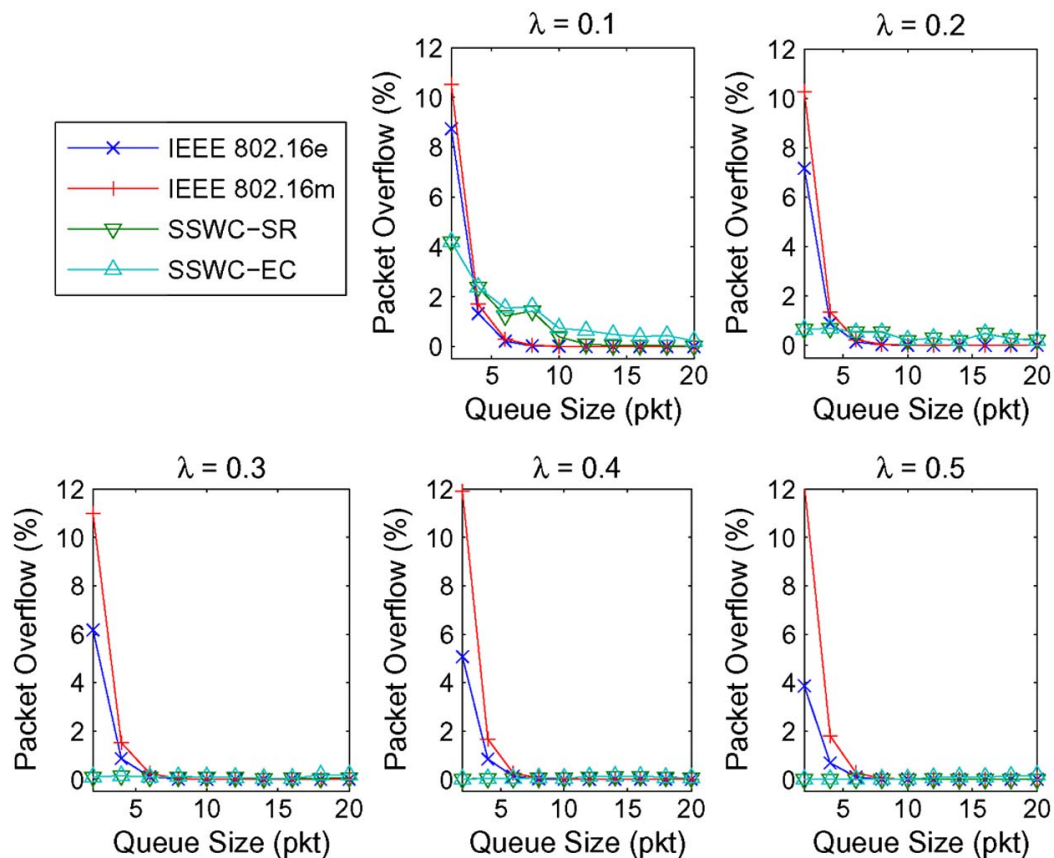

Fig. 11. Performance comparison of packet overflow among IEEE 802.16e, IEEE 802.16m, SSWC-SR, and SSWC-EC approaches under various queue size considerations.
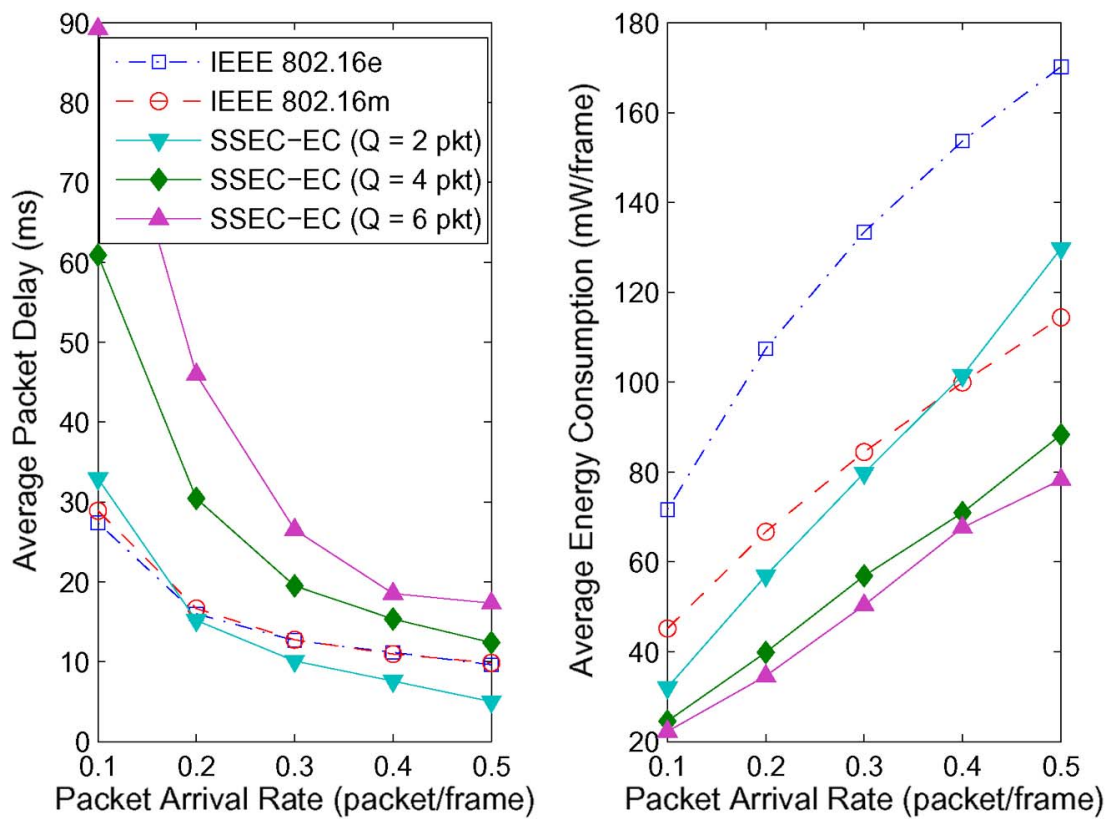

Fig. 12. Performance comparison among IEEE 802.16e, IEEE 802.16m, and SSWC-EC approaches with different queue size considerations $Q$.

queue size, which maximizes the energy conservation of the MS. With the considerations of both tolerable delay and queue size, an efficient sleep-mode operation for mobile BWNs can be acquired by adopting the proposed SSWC approach.

\section{REFERENCES}

[1] IEEE Standard for Local and Metropolitan Area Networks—Part 16: Air Interference for Fixed Broadband Wireless Access Systems, IEEE Std. 802.16-2004, Oct. 2004

[2] IEEE Standard for Local and Metropolitan Area Networks-Part 16: Air Interference for Fixed and Mobile Broadband Wireless Access Systems, Amendment 2: Physical and Medium Access Control Layers for Combined
Fixed and Mobile Operation in Licensed Bands and Corrigendum 1, IEEE Std. 802.16e-2005, Feb. 2006.

[3] K. Yang, S. Ou, H.-H. Chen, and J. He, "A multihop peer-communication protocol with fairness guarantee for IEEE 802.16-based vehicular networks," IEEE Trans. Veh. Technol., vol. 56, no. 6, pp. 3358-3370, Nov. 2007.

[4] M. Aguado, O. Onandi, P. S. Agoustin, M. Higuero, and E. J. Taquet, "WiMax on rails," IEEE Veh. Technol. Mag., vol. 3, no. 3, pp. 1556-6072, Sep. 2008.

[5] K. Etemad, "Overview of mobile WiMAX technology and evolution," IEEE Commun. Mag., vol. 46, no. 10, pp. 31-40, Oct. 2008.

[6] Y. Xiao, "Energy saving mechanism in the IEEE 802.16e wireless MAN," IEEE Commun. Lett., vol. 9, no. 7, pp. 595-597, Jul. 2005.

[7] Y. Zhang and M. Fujise, "Energy management in the IEEE 802.16e MAC," IEEE Commun. Lett., vol. 10, no. 4, pp. 311-313, Apr. 2006. 
[8] L. Kong and D. H. K. Tsang, "Performance study of power saving classes of type I and II in IEEE 802.16e," in Proc. IEEE Conf. LCN, Tampa, FL, 2006, pp. 20-27.

[9] K. Han and S. Choi, "Performance analysis of sleep mode operation in IEEE 802.16e mobile broadband wireless access systems," in Proc. VTC-Spring, Melbourne, Australia, 2006, pp. 1141-1145.

[10] Y.-P. Hsu and K.-T. Feng, "Performance modeling of power saving classes with multiple connections for broadband wireless networks," in Proc. IEEE WCNC, Las Vegas, NV, 2008, pp. 1477-1482.

[11] J. Xiao, S. Zou, B. Ren, and S. Cheng, "An enhanced energy saving mechanism in IEEE 802.16e," in Proc. IEEE GLOBECOM, San Francisco, CA, 2006, pp. 1-5.

[12] M. Peng and W. Wang, "An adaptive energy saving mechanism in the wireless packet access network," in Proc. IEEE WCNC, Las Vegas, NV, 2008, pp. $1536-1540$

[13] M.-G. Kim, M. Kang, and J. Choi, "Remaining energy-aware power management mechanism in the 802.16e MAC," in Proc. IEEE CCNC, Las Vegas, NV, 2008, pp. 222-226.

[14] M.-G. Kim, J. Choi, and M. Kang, "Adaptive power saving mechanism considering the request period of each initiation of awakening in the IEEE 802.16e system," IEEE Commun. Lett., vol. 12, no. 2, pp. 106-108, Feb. 2008.

[15] T.-C. Chen and J.-C. Chen, "Extended maximizing unavailability interval (eMUI): Maximizing energy saving in IEEE 802.16e for mixing type I and type II PSCs," IEEE Commun. Lett., vol. 13, no. 2, pp. 151-153, Feb. 2009.

[16] M.-G. Kim, J. Choi, and M. Kang, "Scheduled power-saving mechanism to minimize energy consumption in IEEE 802.16e systems," IEEE Commun. Lett., vol. 12, no. 12, pp. 874-876, Dec. 2008.

[17] IEEE 802.16m System Description Document, IEEE C802.16m-08/003r8, Apr. 2009

[18] IEEE 802.16m System Requirements, IEEE C802.16m-08/002r8, Jan. 2009.

[19] A. C. Cassandra, "Exact and approximate algorithms for partially observable Markov decision processes," Ph.D. dissertation, Brown Univ., Providence, RI, May, 1998.

[20] L. P. Kaelbling, M. L. Littman, and A. R. Cassandra, "Planning and acting in partially observable stochastic domains," Artif. Intell., vol. 101, no. 1/2, pp. 99-134, May 1998.

[21] A. Andersen and B. Nielsen, "A Markovian approach for modeling packet traffic with long-range dependence," IEEE J. Sel. Areas Commun., vol. 12, no. 5, pp. 719-732, Jun. 1998

[22] T. Yoshihara, S. Kasahara, and Y. Takahashi, "Practical time-scale fitting of self-similar traffic with Markov-modulated Poisson process," Telecommun. Syst., vol. 17, no. 1/2, pp. 185-211, 2001.

[23] A. Nogueira, P. Salvador, R. Valadas, and A. Pacheco, "Fitting self-similar traffic by a superposition of MMPPs modeling the distribution at multiple time scales," IEICE Trans. Commun., vol. E87-B, no. 3, pp. 678-688, Mar. 2004

[24] H. Kobayashi and B. L. Mark, System Modeling and Analysis: Foundations of System Performance Evaluation. Upper Saddle River, NJ: Prentice-Hall, 2009.

[25] C.-H. Ng and B.-H. Soong, Queueing Modelling Fundamentals: With Applications in Communication Networks, 2nd ed. Hoboken, NJ: Wiley, 2008.

[26] Datasheet: SQN1130 System-on-Chip (SoC) for WiMAX Mobile Stations, Sequans Commun., Paris, France, 2007.

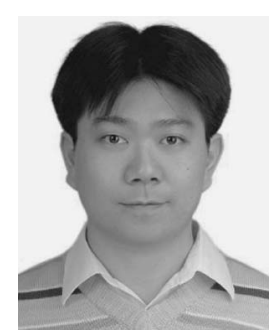

Chung-Hsien Hsu (S'07-M'10) received the Ph.D. degree in communications engineering from $\mathrm{Na}-$ tional Chiao Tung University, Hsinchu, Taiwan, in 2009.

Between May and November 2008, he was with MediaTek Inc., Hsinchu, as an Engineer, working on the design of medium-access control protocols for the IEEE $802.16 \mathrm{~m}$ standard. Since December 2009, he has been with the Industrial Technology Research Institute, Hsinchu, as an Engineer. His current research interests include mobile ad hoc networks, broadband wireless networks, and intelligent transportation systems.

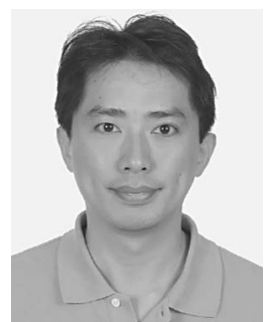

Kai-Ten Feng (M'03) received the B.S. degree from National Taiwan University, Taipei, Taiwan, in 1992 the M.S. degree from the University of Michigan, Ann Arbor, in 1996, and the Ph.D. degree from the University of California, Berkeley, in 2000.

Since August 2007, he has been with the Department of Electrical Engineering, National Chiao Tung University, Hsinchu, Taiwan, as an Associate Professor. He was an Assistant Professor with the same department between February 2003 and July 2007. He was with OnStar Corporation, a subsidiary of General Motors Corporation, as an In-Vehicle Development Manager/Senior Technologist between 2000 and 2003, working on the design of future Telematics platforms and in-vehicle networks. His current research interests include cooperative and cognitive networks, mobile ad hoc and sensor networks, embedded system design, wireless location technologies, and intelligent transportation systems.

Dr. Feng was the recipient of the Best Paper Award at the IEEE Vehicular Technology Conference Spring 2006, which ranked his paper first among the 615 accepted papers. He has served on the technical program committees of the IEEE Vehicular Technology Conference, the IEEE International Conference on Communications, and the IEEE Asia-Pacific Conference on Wearable Computing Systems. He was also the recipient of the Outstanding Young Electrical Engineer Award from the Chinese Institute of Electrical Engineering in 2007.

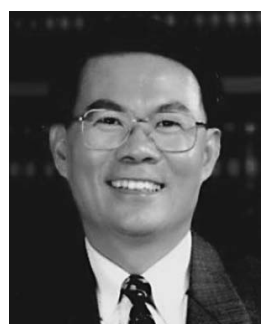

Chung-Ju Chang (F'06) was born in Taiwan in August 1950. He received the B.E. and M.E. degrees in electronics engineering from National Chiao Tung University, Hsinchu, Taiwan, in 1972 and 1976, respectively, and the Ph.D. degree in electrical engineering from National Taiwan University, Taipei, Taiwan, in 1985.

From 1976 to 1988, he was with Telecommunication Laboratories, Directorate General of Telecommunications, Ministry of Communications, Taiwan, as a Design Engineer, Supervisor, Project Manager, and then Division Director. He also acted as a Science and Technical Advisor for the Minister of the Ministry of Communications from 1987 to 1989 . In 1988, he joined the Faculty of the Department of Communication Engineering, College of Electrical Engineering and Computer Science, National Chiao Tung University, as an Associate Professor. He has been a Professor since 1993 and a Chair Professor since 2009. He was Director of the Institute of Communication Engineering from August 1993 to July 1995, Chairman of the Department of Communication Engineering from August 1999 to July 2001, and Dean of the Research and Development Office from August 2002 to July 2004. In addition, he was an Advisor for the Ministry of Education to promote the education of communication science and technologies for colleges and universities in Taiwan during 1995-1999. His research interests include performance evaluation, radio resources management for wireless communication networks, and traffic control for broadband networks.

Dr. Chang is a member of the Chinese Institute of Engineers and the Chinese Institute of Electrical Engineers. He is acting as a Committee Member of the Telecommunication Deliberate Body, Taiwan. Moreover, he once served as an Editor of the IEEE Communications Magazine and an Associate Editor of the IEEE TRANSACTIONS ON VEHICULAR TECHNOLOGY. 